\title{
Photovoltaic Cell: Optimum Photon Utilisation
}

\author{
Thomas Nommensen" ${ }^{1, *}$, Toan Dinh ${ }^{2}$, Liam Caruana $^{3}$, Dennis Tran ${ }^{4}$, Robert McCormick $^{5}$
}

1 E-Mail: Thomas.Nommensen@student.uts.edu.au

2 E-Mail: The.T.Dinh@student.uts.edu.au

3 E-Mail: Liam.Caruana@student.uts.edu.au

4 E-Mail: Dennis.Tran@student.uts.edu.au

5 E-Mail: Robert.McCormick@student.uts.edu.au

*Author to whom correspondence should be addressed; Thomas.Nommensen@student.uts.edu.au

DOI: http://dx.doi.org/10.5130/pamr.v3i0.1409

\begin{abstract}
In the 21st century, global energy consumption has increased exponentially and hence, sustainable energy sources are essential to accommodate for this. Advancements within photovoltaics, in regards to light trapping, has demonstrated to be a promising field of dramatically improving the efficiency of solar cells. This improvement is done by using different nanostructures, which enables solar cells to use the light spectrum emitted more efficiently. The purpose of this meta study is to investigate irreversible entropic losses related to light trapping. In this respect, the observation is aimed at how nanostructures on a silicon substrate captures high energy incident photons. Furthermore, different types of nanostructures are then investigated and compared, using the étendue ratio during light trapping. It is predicted that étendue mismatching is a parasitic entropy generation variable, and that the matching has an effect on the open circuit voltage of the solar cell. Although solar cells do have their limiting efficiencies, according to the Shockley-Queisser theory and Yablonovitch limit, with careful engineering and manufacturing practices, these irreversible entropic losses could be minimized. Further research in energy losses, due to entropy generation, may guide nanostructures and photonics in exceeding past these limits.
\end{abstract}

Keywords: Photovoltaic cell; Shockley-Queisser; Solar cell nanostructures; Solar cell intrinsic and extrinsic losses; entropy; étendue; light trapping; Shockley Queisser; Geometry; Meta-study 


\section{Introduction}

\subsection{Black body}

The purpose of a photovoltaic cell is to produce useable energy, from the solar spectrum of the sun by absorbing emitted radiation [1]. In principle, the sun can be modelled as a black body, which emits a spectrum characteristic of its temperature $[2,3,4]$. The concept of a black body can be extended and generalized to every materialistic body, which is in thermal equilibrium $[2,5]$.

This phenomenon was formulated correctly by Planck, where the assumption was that energy was quantized, which he used to fit the emitted spectrum of a blackbody [2,6]. The formulation resolved the blackbody problem and thus marked the end of the era of classical mechanics [6]. From Figure 1.1. The differences between the Classical model and the Quantum model are evident.

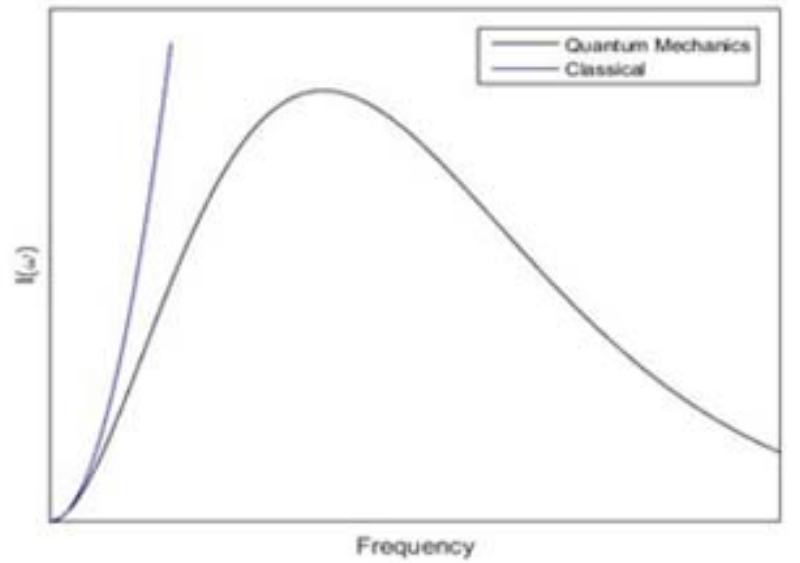

Figure 1.1. The difference in blackbody intensity between Quantum and Classical formulation. The body is at a temperature of $6000 \mathrm{k}$

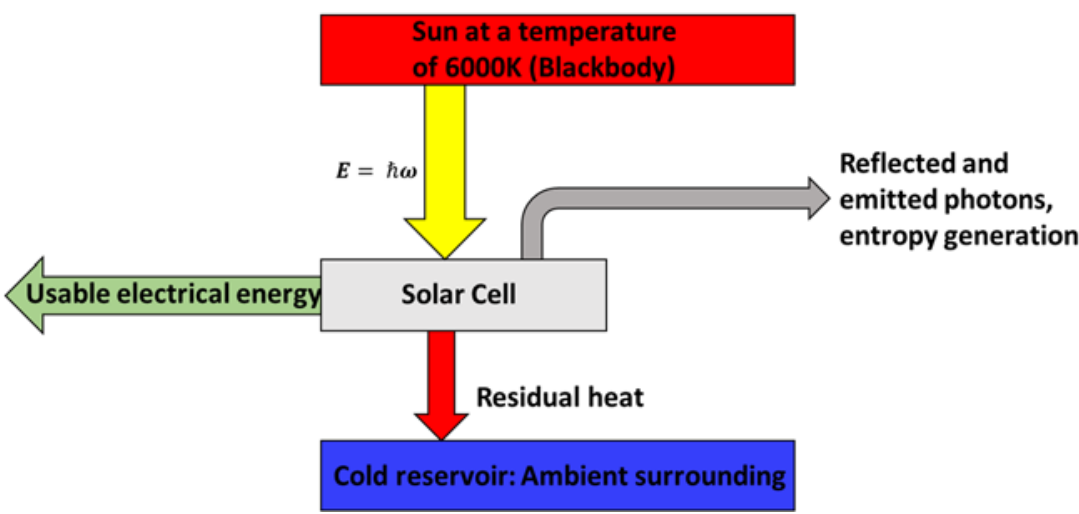

Figure 1.2. A schematic of the energy flow in a solar cell. The sun emits blackbody radiation (photons). The solar cell converts photons into electrical energy for photons above energy bandgap.

A blackbody can be formally defined as:

A black body appears to be black as it absorbs the incoming electromagnetic wave, but reemits it at another frequency, characteristic of its temperature. The colder the body, the lower the associated brightness and the longer the wavelength, of the re-radiated electromagnetic wave. When the body is not in thermal equilibrium with another body, the emission and absorption process is irreversible and is characterized as a thermodynamic fluid, with which, it transmits thermodynamic parameters such temperature, energy, volume, entropy and pressure [2, 5, 3].

The above generalization of a blackbody is present in all bodies regardless of their physical state. Black body radiation also occurs in solar cells, as it absorbs incoming electromagnetic waves which are quantized and carry the energy $E=\hbar \omega[2,3,7]$. The emitted radiation by the sun is absorbed by a solar cell to employ the photoelectric effect, which converts incoming photons into useful work [1]. Therefore, it is of essential importance to capture and trap photons of higher energy inside a semiconductor material using nanostructures, as to minimize photon loss and achieve maximum efficiency. Since every semiconductor material has a characteristic bandgap, such as Silicon $(1.11 \mathrm{eV})$, only a certain amount 
of photons are able to produce a photoelectric current. For the photoelectric current to occur, a photon is required to an energy $E_{g}=\hbar \omega_{g}$ or greater than the bandgap of the semiconductor [3].

\subsection{Entropy}

All heat engines that produce some form of energy, which can be used to do work, are sources of entropy formation $[2,6,8,9]$. The concept of a heat engine also applies to solar cells, which employ the use of photons emitted by a black body $[1,3,10]$. It is vital to consider the entropic evolution of the system's parameters, as an increase in entropy tends to degrade energy conversion and efficiency [1, 3 , $5,11]$.

Entropy is a state function, independent of the past and the path taken to achieve the systems current state $[2,6,8,9]$. The conceptual manifestation of entropy serves as an explanation as to why certain events spontaneously occur and others do not such as the propagation of time $[2,5,8,9]$. The second law of thermodynamics is a description of the dispersion of energy and the disorganization of matter [2, $8,9]$. From the first law of thermodynamics, energy can neither be created nor destroyed and it is a conservative quantity; this is however not the case with entropy [5, 6]. Entropy and energy are very similar quantities as they both are extensive state variables which are only determined relative to a reference state [5]. They, however, differ, in the aspect that unlike energy, entropy is a non-conservative quantity, and has the ability to stay constant or increase, even in an isolated system, but never spontaneously decrease $[2,5,6,8,9]$.

The first statement that entropy can stay constant is an idealized case where a system is taken from state "a" to another state " $b$ " using some pathway and during this procedure no energy is lost. If this case is true, then propagating in the reverse direction would output the same energy which was applied. In this idealization, the process takes a constant entropy value and is labelled a reversible process. If energy is lost during this process, entropy is not conserved but increasing due to dissipation of energy [2, 5, 6 , $8,9]$. In all real heat engine systems, dissipation of energy always occurs and is parasitic, as it degrades energy [5,6]. Entropy takes its mathematical form as:

$$
\Delta S=S\left(V_{b}, T_{b}\right)-S\left(V_{a}, T_{a}\right)=\int_{a}^{b} \frac{d Q}{T}
$$

The quantity " $d Q$ " is energy being transferred as reversible heat, and the quantity " $\mathrm{T}$ " is the temperature, at which this exchange of energy is occurring. " $\Delta S$ " is the change in entropy of the system as it proceeds from state "a" to state "b", and is a function of both, an extensive variable (Volume) and an intensive state variable (Temperature) $[5,6,8]$.

Armed with the second law of thermodynamics, it possible to justifiably demonstrate certain properties of entropy:

Entropy is an extensive variable, which is additive and increases as time progresses. It is a state function in the sense that it does not depend on the path taken, but only depends on the initial and final states of the system, unlike energy and work which demonstrate path dependency. Furthermore, entropy 
is a non-conservative quantity that dictates the spontaneity of events. If the change in entropy is positive the event is spontaneous, however if the change is negative, the event is not spontaneous. The quantity of entropy is a measurement of the joule per kelvin [2, 5, 6, 8,9].

This case is only possible if work is being done on the system, hence a negative entropy change. It, however, does not violate the second law of thermodynamics because, wherever there is a decrease in entropy, there is always a greater entropy increase as to create a net increase in entropy of the universe $[2,6]$.

Solar cells are inherently comparable to the concept of heat engines [1, 10]. Since it is impossible to generate work from two reservoirs which are in thermal equilibrium with each other, solar cells require an external heat reservoir, namely the sun $[1,2,6,11]$. In a heat engine context, the sun is the heat reservoir and has a temperature $\mathrm{T}_{\mathrm{s}}$, the cold reservoir is the solar cell itself and is in thermal equilibrium with its ambient temperature, $\mathrm{T}_{\mathrm{A}}$. From the Blackbody section, the sun can be modelled as a blackbody and hence emits radiation characteristic of its temperature. The emitted black body radiation can be considered as a stream of energy packets, namely photons. These photons are then absorbed by the solar cells to produce useful work using the photoelectric effect $[1,10]$. As the solar cell absorbs the incoming photons with energy, $E=\hbar v$, the solar cell begins to generate heat, as a result of photon cooling [1, 3]. As photons can be both considered as a particle and a wave, it is therefore describable as a gas, instead of using volume the concept of étendue is applied.

As étendue increases, so does the photon cooling effect which increases the entropy of the solar cell $[1,10]$. The photon cooling effect is analogous to the expansion of a gas, as the étendue increases, so does the photons apparent 'volume' increase, which describes an increase in the photon modes in which it can occupy $[1,10]$. Therefore, it is essential to match absorption and emission angles of the solar cell, as to avoid unnecessary entropy generation $[3,11]$. Another parasitic entropy formation parameter is the current produced by the solar cell which degrades the amount of work available per photon. Another parasitic entropy formation parameter is the current produced by the solar cell, which degrades the amount of work available per photon.

Due to the solar cell being a multitude of small systems, its total entropy generation is simply the sum of all the internal systems, as dictated by the additive property of entropy:

$$
S_{\text {total }}=S_{1}(i)+S_{2}(i)+\cdots+S_{n}(i)=\sum_{n=1}^{n} S_{n}(i)
$$

To increase the solar cells overall efficiency, it is of great importance to minimize each entropic generation subsystem, as much as possible.

\section{3. Étendue}

In any optical system such as a solar cell, a fundamental physical law, called étendue, needs to be taken into consideration in order to increase efficiency $[3,12,13]$. The term étendue is often considered 
in geometrical and non-imagining optics and is closely related to entropy, which gives rise to a boundary condition for the enhancement of optical paths $[3,13,14,15]$.

Étendue is the measure of incoming beam properties, such as angular spread and size; however it can also be interpreted and applied in different contexts. In the thermodynamic context, étendue is the optical measure of entropy, but can also be viewed as volume in correspondence to a volume element in phase space, with wave vector components $[10,12,15]$. Étendue can be viewed as a volumetric measurement and from early quantum mechanics; it can be understood as the measurement of quantum states, which a photon can occupy during optical propagation of a medium $[10,15]$. This concept of quantum states of a photon in a volume results in a link to thermodynamics, due its statistical nature [15]. However, in geometrical optics, étendue describes a systems ability to emit or accept light $[15,16]$. In the absence of any scattering or absorption, étendue is a conservative property, due to the invariance of the volume in phase space and its conserved quantum states $[10,15]$. This inherently derives from the second law of thermodynamics and quantum mechanics $[10,13,14,15]$.

The close relation of Étendue with entropy is apparent when considering the presence of scattering and absorption. When a beam of light is scattered or absorbed its étendue can increase due to photon losses during its propagation. The relation between entropy and étendue is justified in the sense that randomization of photons is a thermodynamically irreversible property [15]. This leads to the generalization of the étendue:

The étendue of a light beam, propagating through a medium, can stay constant or increase due to losses but never decrease [11, 12, 13, 15].

In the first case of the theorem, where étendue is conserved, a perfect system is being considered. However, in a real system, the second statement is usually the case [13]. In the event étendue is decreasing, a loss of light occurs and a higher state of order has resulted. As such, a close correlation to entropy, in that it can decrease if energy is lost from a system or energy is used to create order [12, 13, 15]. A violation of the principle of étendue occurs when considering an optical system, in which it is possible to decrease both the beam angle and beam size simultaneously [13].

From geometrical optics, the concept of étendue arises from the idea that a beam, with some form of vectorial direction with a solid angle " $\delta \theta$ " passes perpendicularly through an area element $\delta A$. Forming an element of étendue, $\delta \xi$, given by $[7,10,15,17]$ :

$$
\delta \xi=n^{2} \cos \psi \delta \theta \delta A
$$

Where " $n$ " is the refractive index of the medium, " $\psi$ " is the angle between the vectorial direction of propagation and the normal area element " $\delta A$ ". 
Since every optical system is usually composed of several components, optimisation is important as the component with the lowest étendue sets the limiting value for the entire system [16]. Hence, it is essential to carefully engineer an optical system as to increase efficiency [3]. Therefore, to optimise the system, the étendue of both the source and the system should be closely matched [16].

\subsection{Shockley Queisser}

In 1961, William Shockley and Hans J. Queisser proposed a theoretical limit which all single-junction band gap based solar cells are governed by [4, 18, 19]. Their assumptions are based on the fact that solar cells can be modelled as a black body with a temperature of $300 \mathrm{~K}[12,18]$. Shockley and Queisser furthermore proposed that the theoretical efficiency of a solar cell is limited by several factors, namely: temperature of the sun, temperature of the solar cell and the band gap energy of the semiconductor material $[18,19]$. In majority, entropy sets a significant constraint on the efficiency of solar cells due to the consequence of unavoidable atomic processes $[10,18,19,20]$.

The general idea proposed by Shockley and Queisser was to equate incident photon flux, which have a higher energy than the bandgap, to the photon flux emitted by the solar cell plus the electrical current generated by those photons $[10,17,18]$. With this principle, it is evident that only a fraction of the photons incident are able to be converted into usable electrical energy at thermal equilibrium [10, 18]. The relation of this is given as:

$$
\Phi_{\text {in }}=\Phi_{\text {out }}+I / q
$$

Where " $\Phi_{\text {in }}$ " and " $\Phi_{\text {out }}$ "is the incoming and re-radiated photon flux respectively. " $I$ " represents the current drawn from the solar cell and " $q$ " is the electronic charge. The explanation of the solar cell radiating photon flux out is due to other factors, such as radiative recombination of electron-hole pairs which are dependent on the minority carrier's lifetime [18, 19]. Furthermore, some of the incident photons, which have a greater energy than the bandgap, do not create electron-hole pairs and must therefore be viewed as a probabilistic event [18].

Shockley and Queisser further considered other aspects of the solar cell which contribute to loss of efficiency, namely the angle of incidence which is a geometrical factor, the ability to remove holes and electrons from their respective regions and the absorption coefficient for solar energy striking the surface $[10,18,19]$. From the paper, they concluded that the maximum efficiency for a semiconductor with an energy gap of $1.11 \mathrm{eV}$ such as silicon, is $44 \%$.

The Shockley and Queisser limit has been used extensively in research areas concerning photonic structures as a benchmark [4]. The remarkable property of the limit was that it described the efficiency of solar cells, employing only the incident and emitted light beams [10], both of which are functions of the solid angle subtended by the sun and the properties of the cell's structure $[10,19,21]$. 
By extension of the Shockley and Queisser limit, the electron and hole quasi - Fermi levels, $q V_{O C}$ are approximated as $[11,19]$ :

$$
q V_{O C}=\left(1-\frac{T_{O}}{T_{S}}\right) E_{g}+k_{B} T_{O} \ln \left(\frac{T_{S}}{T_{O}}\right)-k_{B} T_{O} \ln \left(\frac{\xi_{\text {out }}}{\xi_{\text {in }}}\right)-k_{B} T_{O} \ln \left(\frac{I_{l}}{I_{l}+I}\right)
$$

Where " $V_{O C}$ " is the open circuit voltage, " $\xi$ " is the étendue of the sun and the emitted radiation, " $T_{O}$ " and " $T_{S}$ " are the temperatures associated with the cell and the sun, " $E_{g}$ " is the band gap energy and " $I_{l}$ " is the photo generated current $[10,20]$.

Equation 5 can not only be used to calculate the open circuit voltage, but it can be of use in determining parasitic entropic losses caused by improper engineering [19, 20]. The first part of the equation represents the Carnot efficiency of converting a photon with energy $E_{g}=h \omega$ to electrostatic energy [19]. The second term is the irreversible entropy generation associated with photon cooling [10, 20]. The third term represents the étendue increase, which proves to be the one of the most fundamental energy losses $[10,19,20]$. The last term in the equation is the entropic loss due to current extraction from the cell [20]. Another term which should be notably added to the equation is the Quantum Efficiency and is dependent on the interface of surface manufacturing, but this term is usually taken to be unity $[10,19]$.

Aside from p-n junction engineering, a promising area which also demonstrates theoretically that the Shockley Queisser limit can be exceeded for non-concentrating sun-light, by photonic light trapping [21]. Light trapping enables radiative losses to be reduced greatly near photon energies just above the band gap, as these energies are poorly absorbed by the junctions [19, 21]. It is evident that the Shockley and Queisser limit can be exceeded, by employing optimal engineering of light trapping systems and p$\mathrm{n}$ junction technology $[11,21]$.

\subsection{The Yablonovitch limit}

Current developments in light trapping regimes often use the Yablonovitch limit, sometimes referred to as the $4 \mathrm{n}^{2}$ limit, where $\mathrm{n}$ is the refractive index. The limit is used as a useful benchmark to test developing technological advancements [22]. The Yablonovitch limit is used to describe a thermodynamic limit in regards to geometrical optics using the assumptions that the medium is a weak absorber and that perfect diffusion of photons occurs, regardless of the angle of incidence [4, 22, 23, 24]. The reasoning behind the assumption that photons are perfectly randomized in a medium, is due to internal reflection of textured surfaces from the back and front interfaces or other random events, such as light source movement $[4,23,24]$. The randomization of photons is not only exclusive for completely unordered surfaces, but also observable for an ordered textured surface [24].

Using the Planck distribution and group velocity of the wave, it can be seen that the intensity inside the medium is $n^{2}$ times larger. As such the Yablonovitch limit is given by: 


$$
l_{\text {eff }}=\frac{4 n^{2} w}{\sin ^{2} \theta}
$$

Where $l_{\text {eff }}$ is the path length of the photon travelling, " $w$ " is the thickness of the medium and " $\theta$ " is the angle of the cone in which the photon can leak out of the light trapping medium $[4,24,25]$. This equation usually is used in the case where $\theta \approx \frac{\pi}{2}$ so the denominator is simply one [25]. It can be concluded that the Yablonovitch limit is a thermodynamic limit in terms of the maximum path length enhancement possible for a photon inside a medium [4]. When further inspecting the resulting equation, the $n^{2}$ term can be explained using the density of photon states which it can occupy in the medium [4, 24].

The $4 n^{2}$ factor can be used to increase the absorption of photons in the material and hence, increase the efficiency of a solar cell [4, 22, 23]. However, the assumptions made by Yablonovitch are no longer valid at scales where the thickness of the material are comparable or less than the wavelength of the incident light $[4,15,25]$. Current simulations and experimental evidence suggests that nanophotonic structures are able to exceed the Yablonovitch limit due to their small dimensions $[4,22,25]$. The Yablonovitch limit is however, observable at the large scale regime where the structure's dimensions are far greater than the impingent photon spectrum $[15,25]$.

\subsection{Light Trapping Techniques}

Light trapping is achieved by harnessing total internal reflection of the incident light inside the nanostructures of the cell. This allows for more efficient and the potential for thinner devices to be manufactured $[14,23]$.

When the light hits the surface at an angle greater than the critical angle the light will be partially internally reflected [26]. The reflected light will collide with another surface where the same process repeats. Therefore, a low $\alpha$ angle is most desirable in order to reduce the amount of reflected light [24, 26].

The randomization of the propagation of light is of great importance in photovoltaic cells as it allows for photons to be absorbed with energies near the bandgap of the silicon semiconductor [14, 19]. As scattering inside a medium increases, the photons probability to interact with geometrical surfaces, which are etched into the silicon substrate, increases [27, 28]. Not only do geometrical structures increase the scattering process, they also promote a reduction in the material used which results in lower manufacturing costs [20]. 


\subsubsection{Nanopillars and Nanowires (Figures 1.6.1.1 and 1.6.1.2)}

For light trapping to be possible, different geometries are required to scatter the incident photons [25, 29]. The geometry of nanopillars and nanowires are vital in current research regarding light trapping structures. Both nanopillars and nanowires exhibit a cylindrical geometry, which are able to trap incident photons a certain angle, labelled as " $\alpha$ " [30, 29]. When a photon exceeds the critical angle, it starts to reflect back and forth from the adjacent nanostructures [29, 32, 33]. This back and forth reflection is often referred to as the Fabry Perot interferometer. This resonation of the photon is a very characteristic of those nanostructures and enable efficient light trapping [26, 33].

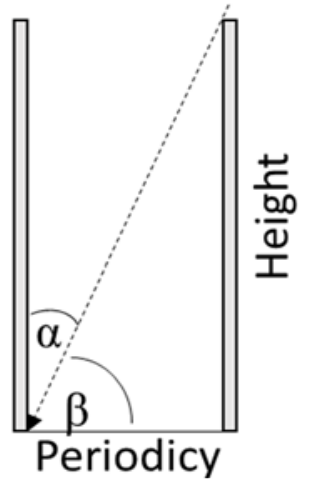

Figure 1.6.1.1. This Figure portrays incident photons on a nanowire which are at the critical angle $\alpha$. Angles greater than the critical angle will result in secondary reflection.

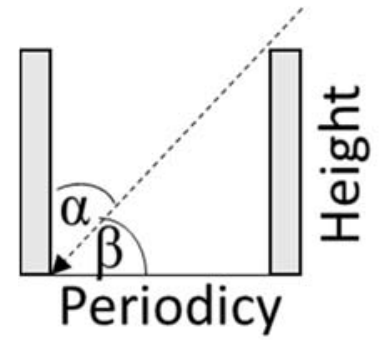

Figure 1.6.1.2. In this Figure the incident photons are encountering a nanopillar. The critical angle is labelled as $\alpha$.

\subsubsection{Nanocones and Pyramidal structures (Figure 1.6.2)}

Nanocones and Pyramidal structures exhibit a close relation in that when observed from the side they have an identical geometry. Nanocones have an all-round smooth surface with no corners $[10,35,36]$. Pyramidal structures have corners and only four smooth surfaces from which light can be reflected. At a certain incident, angle light will be trapped between adjacent structures [36, 38]. This induces light scattering and therefore enhancement of photons infusing into the semiconductor to generate electron hole pairs [4].
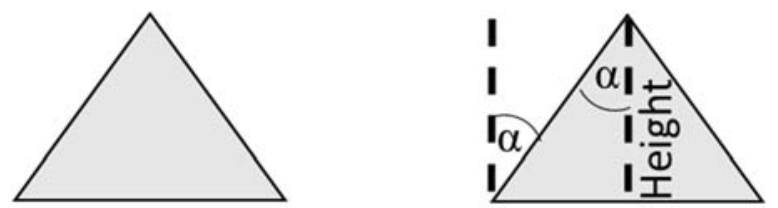

\section{Periodicy}

Width

Figure 1.6.2. Light trapping of photons within a nanocone. The angle which the cone makes with the substrate is labelled as $\alpha$.

\subsubsection{Nanodomes (Figure 1.6.3)}

The nanodome geometry is fairly unique, in the sense that they do not cause incident photons to resonate between adjacent structures as much as the nanowires or nanopillars [34, 33]. These geometries, 
generally speaking, cause light to be scattered into the surrounding medium which then minimizes the chance of the photon to be able to return to its original trajectory [23, 29].

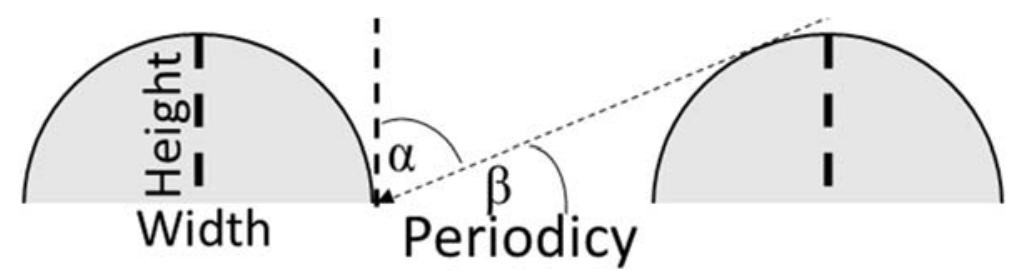

Figure 1.6.3. The Figure displays the critical angle $\alpha$ of the nanodome structure. A photon incident greater than the critical angle will result in secondary reflection.

\subsubsection{Improvements of Light Trapping}

Since etched geometries promote photon scattering, it is important to note that some geometries cause the photons to only change direction [19]. Therefore, Snell's law will be of great assistance in the ability to further trap photons. This is due to the fact that light, which is travelling from a high index medium to a low index, is susceptible to total internal reflection. Employing Snell's law enables the minimization of the cone from which the photons can escape.

Additionally, to avoid photons from escaping, Transparent Conducting Oxide layers (TCO) may be used to allow refracting index matching which evidently reduces étendue formation. Using TCO (Figure 1.6.4) enables high transparency for photons in the solar spectrum and high mobility for charge carriers [38]. This is similar to the use of angular selective filters which allow photons to leave the medium only at very certain angles [38]. All these techniques increase the photons effective path length and probability of interacting with the

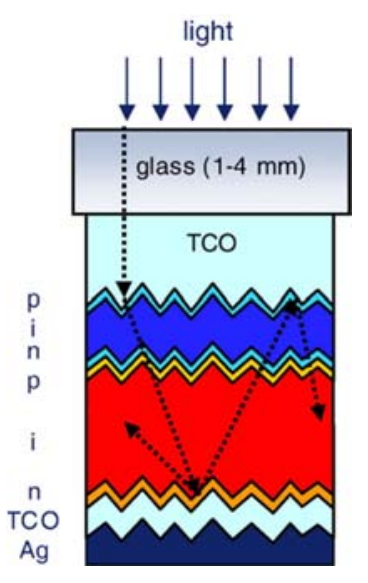

Figure 1.6.4. Sketch of $\mathrm{Si}$ tandem Solar cell, W. Beyer 2007 semiconductor [37].

\subsection{Literature Review}

As a part of the meta-study, reviewing and examining research is required to grasp the concepts of light trapping but also experimental data in which then comparable data can be mapped out. In this respect, the literature review is organised into categories of nanostructures where other groups have experimentally done. Drawing from this, it outlines the possible increases in current that these nanostructures exhibit which are not from the initial incident ray.

\subsubsection{Nanocones}

By implementing a double-sided grating nanocone onto a photovoltaic cell, the antireflection and light trapping capabilities can be improved [31, 30,32]. The experiment conducted by the Stanford Institute for Materials and Energy Sciences found that it is possible to produce a short circuit current of $34.6 \mathrm{~mA} / \mathrm{cm}^{2}$ for a cell thickness of $2 \mu \mathrm{m}$. With the analysis of the paper, it can be observed that the short circuit current reaches close to the Yablonovitch limit, which within the experiment was measured to be at $35.5 \mathrm{~mA} / \mathrm{cm}^{2}$ [31]. 
Besides the geometry of nanocones, the contributors for this light absorption increase is due to the grated surfaces derived from the anti-reflection properties of the nanocones, the light absorption from texturing of the Si thin-film cell surfaces and the geometry of nanocones [30, 32].

The double-sided grating is fitted on each side of the cell, and is essential in improving the efficiency of the system. The front surface grating was used to improve the antireflection capabilities of the cell, whilst the back surface is primarily used to trap the light [31]. When the grating was tested separately, the back surface grating achieved a short circuit current of $30.5 \mathrm{~mA} / \mathrm{cm}^{2}$, whereas the top surface grating achieved a current of $31.7 \mathrm{~mA} / \mathrm{cm}^{2}$. All of these gratings compared to a control solar cell (plain flat thin film solar cell) shows dramatic improvements as the cell only achieved a current of only $19.7 \mathrm{~mA} / \mathrm{cm}^{2}$ [31].

To optimise the antireflection of nanocones, they must be placed into an array that is in the subwavelength regime for the incoming light [29, 32]. This provides a smooth index transition for the light from the air to the silicon and provides more stable photoelectric current compared to non-texture flat film solar cells [30, 31, 32, 33].

\subsubsection{Nano pillars}

Nanopillars are similar in concept and design to nanocones, they however possess different geometries. Due to this nanopillars have different applications and yield different results when integrated into solar cells $[30,31,33]$.

Much like nanocones, the nanopillars must be placed into an array to achieve maximum absorption efficiency [33]. To further enhance the absorption efficiency, a dual-diameter nanopillar structure is developed [33]. Similar to the nanocones, a small diameter tip is implemented to minimise reflectance and the base is given a larger diameter to further maximise effective absorption [31, 33].

\subsubsection{Nanowires}

In the current state nanowires utilises the same aspects of those of nanocones and nanopillars. A study done by the Integrated Systems Laboratory in Zurich, Switzerland, investigated the design features of nanowire photovoltaic cells. It was found that Nanowires constructed with a diameter below a certain threshold will output a low short-circuit current [28]. Even when attempting to compensate for this with higher density nanowiring, there was little that could be done to make up for the lost current [28]. By placing the nanowires further apart than usual and varying this change accordingly, an improvement with regards to the efficiency can be observed [29, 27, 28].

Another study in 2013 done in part by scientists at Lund University and in collaboration with various Swedish scientific institutions showed that a $13.8 \%$ efficiency could be achieved using nanowire technology, with having only $12 \%$ of the cell surface covered [26]. A $\mathrm{V}_{\text {oc }}$ of 0.906 , as well as a conversion of sunlight to photocurrent percentage of $71 \%$ was also achieved [26]. 


\subsubsection{Transparent Conducting Oxide Films (TCO)}

The incorporation of either amorphous or microcrystalline silicon as an absorber material has a critical role in regards to a photovoltaic device's light trapping performance [37, 38].

Using TCOs for effective light trapping requires that the TCO silicon interfaces have a rough TCO surface, placed upon the silicon thin film [37]. The light trapping and scattering mechanism works through increasing the refractive index of the silicon material, in comparison to the TCO material, which in turn, causes the solar cell's light trapping capabilities and cell efficiency to be enhanced [37, 38]. This technology also allows for the cells to be made thinner, therefore reducing cost and materials used [37, 38]. The rough surfaces are obtained through growth of the TCO films onto textured glass, or through chemical deposition texturing techniques [37].

A research group at the Institute of Photovoltaics in Julich, Germany, were able to enhance the light trapping capabilities of a photovoltaic cell, using an Aluminium doped Zinc Oxide film, which achieved an open circuit voltage of $1.43 \mathrm{~V}$ with an efficiency of $15 \%$ [38]. Much like the experiment conducted by Stanford University involving dual-sided grating nanocones, the effectiveness of coating both the front and back surfaces were investigated [38]. TCO films are developed primarily as a front surface contact. A highly reflective back contact is also essential so that the silicon absorber layer, embedded by TCO films, is as effective as possible [37, 38].

\section{Methodology}

The purpose of this meta-study is to compare and analyse different nanostructures of voltaic cells. The study is undertaken by utilising resources that look only at P-N junctions on silicon substrates and how, by changing the nanostructure could alter different parameters including entropy, intrinsic and extrinsic losses.

Since there are outstanding resources on photovoltaics, restrictions are used to assure relevance and integrity. As such, data was restricted by using keywords 'Photovoltaic cell', 'ShockleyQuesisser', 'Solar cell nanostructures', 'Solar cell intrinsic and extrinsic losses', 'entropy', 'étendue', 'light trapping', 'Shockley Queisser', 'Geometry' peer-reviewed sources from Google Scholar and Science Direct are within the last 40 years for theoretical papers and within the last 10 years for experimental data.

By going through multiple references in the found journal articles graphs, tables or raw data, with comparable data was obtained and placed within a table. Using this data, Excel (computer graphing program) was used to make scatter graphs to then compare certain variables over one another. 
The efficiencies of each system can then be analysed from the graph and comparatively demonstrate which nanostructure is therefore more efficient. Following from this, the study will further discuss about how to improve the maximum efficiency of the system in regards to exceeding the Shockley Queisser and Yablonovitch limit.

\section{Results}

\subsection{Geometry of the Light trapping structure}

3.1.1: Critical Angle dependency on height of the geometry

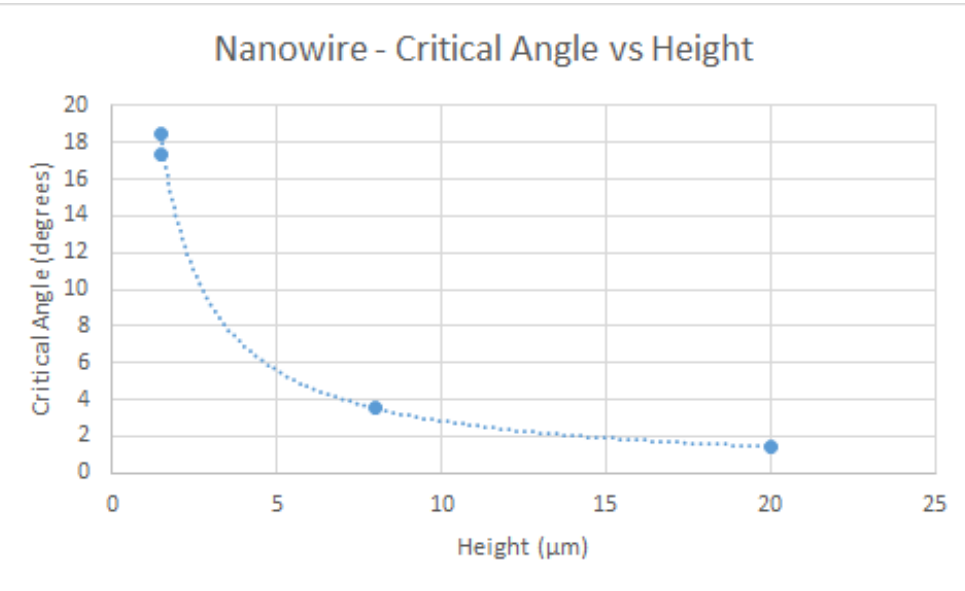

Figure 3.1.1.1. Height and the critical angle exhibited by the Nanowire is compared to other nanowires. [27, 34].

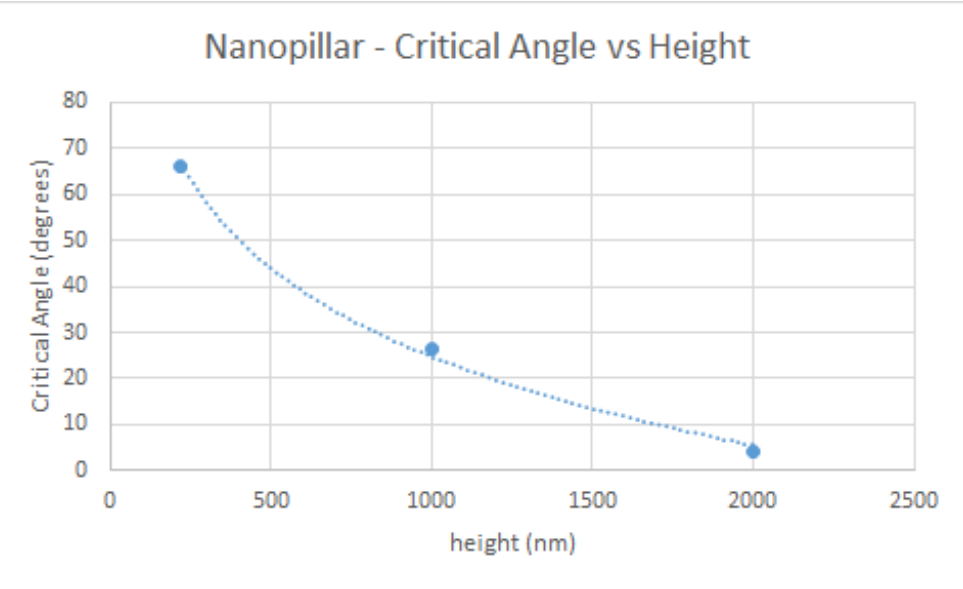

Figure 3.1.1.2. A plot of the different nanopillars which varied in height. [26, 33, 37].

From Figure 3.1.1.1, it is apparent that the $\alpha$ angle is a function of the height of the nanostructure. In this Figure, this nanostructure is a simple nanowire with different heights [37, 38]. It can be seen that the slope of the nanowire is very steep in that a small change in the height has a serious effect of the exhibited critical angle [27, 28, 29]. The exponential nature of the critical angle dependency of the height is also apparent in Figure 3.1.1.2, where the nanopillar plot is also demonstrating an exponentially decreasing nature in the critical angle with respect to the height.

Comparing the two Figures, it is apparent that height of the geometry in general has a great effect on the critical angle. However, the slopes of the two plots appear to be different which demonstrates that there is some form of geometrical shape dependency. The slope of the Nanowire appears to be decreasing at a greater rate when compared to the Nanopillar nanostructure. From the étendue section, it can be seen that a smaller critical angle causes a minimization of the étendue formation, and is formally described as the loss of light. Having a small critical angle promotes photons to be resonating between adjacent nano structures, causing an increase in probability of the photon being absorbed by the semiconductor material. 


\subsubsection{Current dependency on Critical Angle}

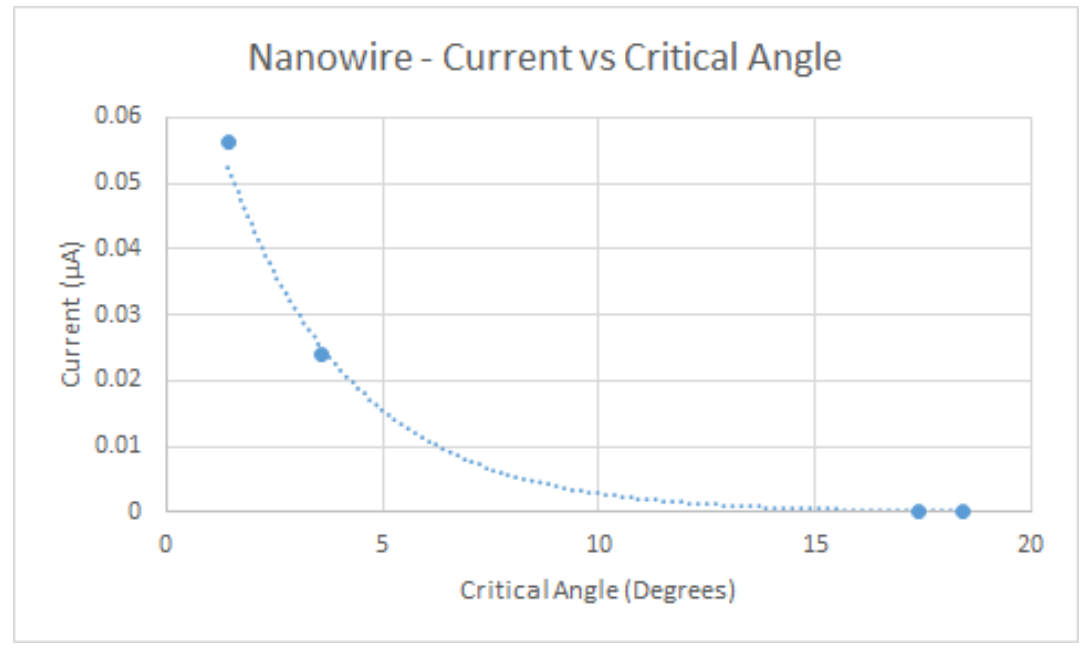

Figure 3.1.2. Plot of various Nanowire current outputs for the same structure with different critical angles [27,34].

The ability of the photon to be absorbed by the semiconductor material is of critical importance, as photons require an equal or higher energy than the bandgap to generate electrons. The critical angle can therefore become crucial in the trapping of high energy photons. When a photon incident on the semiconductor material is being absorbed by the semiconductor material, the electron of the material is being promoted to the conduction band which can then be used to generate a current. Trapping the photon increases the probability of photon absorption in the semiconductor material.

This is being displayed in Figure 3.1.2 where the generated current is a function of the critical angle. It can be seen that the generated current is exponentially declining as the angle increases which can be explained with an increase in étendue where the photons incident are not resonating between the nanowires but instead are escaping the nanostructure. Furthermore, an increase in the critical angle results in a greater probability of the photon to escape and not produce electrons which could be used for electrical power output.

Another issue associated with having a greater critical angle is that with electrons being promoted to the conduction band radiative recombination is a parasitic loss in solar cells. This occurs as Shockley and Queisser discussed that electrons jump from the conduction band to the valence band and recombine with holes to form photons. These photons are then emitted to the material and potentially lost. The employment of a small critical angle forbids this loss of high energy photons by being trapped within the nanostructure. Forming a resonating propagation between adjacent nanowires. 
From the Shockley Queisser limit section another limitation on the light trapping is that the number of incident photons has to equal the number of photons escaping the solar cell structure under thermal equilibrium conditions. This limitation is a result of the quantum states which a photon can occupy in a certain volume of space. It is therefore important to promote the absorption rate of the high energy photons as to increase the current output.

\subsubsection{Current Dependency on Surface Area}

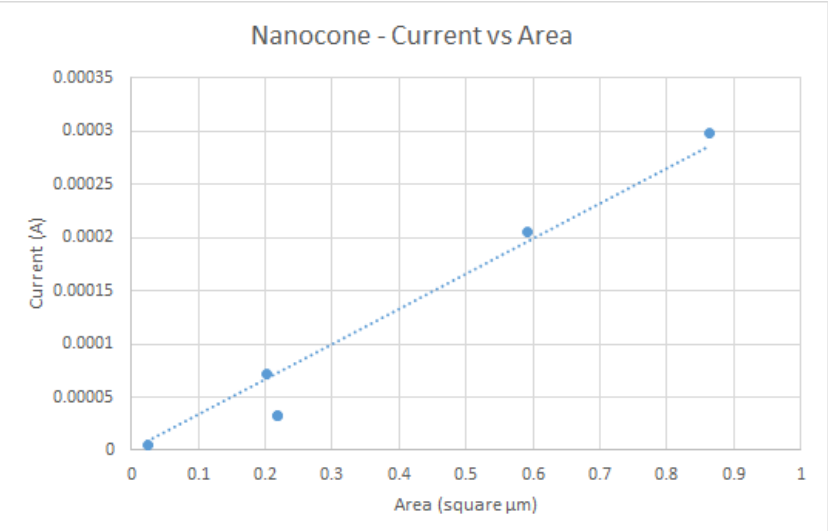

Figure 3.1.3.1. Same structure nanocone current outputs against different surface areas [30, 35, 36, 37].

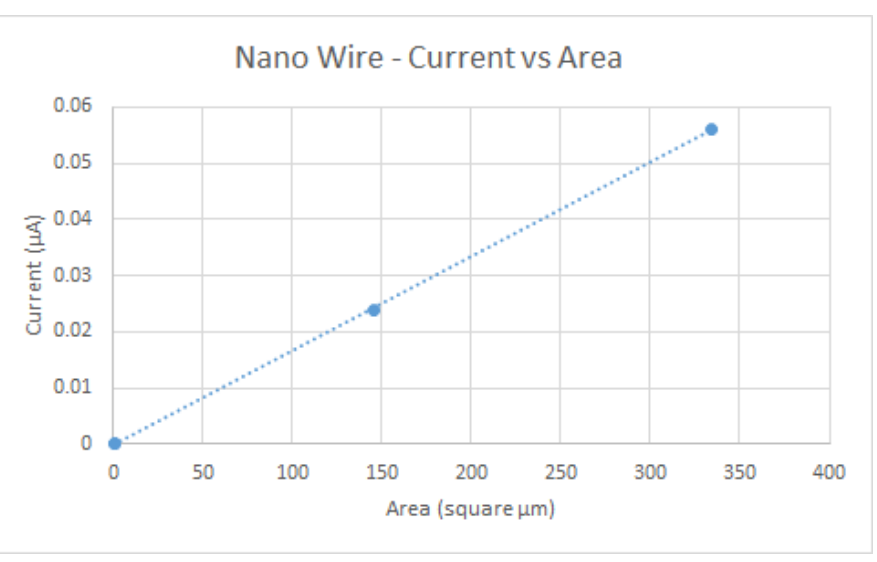

Figure 3.1.3.2. Same structure nanowire current outputs against different surface areas [26, 33, 37].

Figure 3.1.3.1. and Figure 3.1.3.2. both show that an increase in surface area has a significant impact on the output current of the solar cell. A greater surface area is desired because of the way in which it allows more photons to reach the semi-conductor in the cell, therefore increasing the overall output current. The nanocones, when compared to the nanowires, produces a greater effective current when given a similar overall surface area, due to the geometry of the nanocones being better fitted to trapping light. This is also shown in the graphs comparing the critical angle to that of the output current. From the geometry of the nanocones, it is evident that at a certain angle the photon will be reflected off the first structure and reflected out of the solar cell by an adjacent cone, resulting in a reduction of absorption in the cell. Thus, careful engineering of the nanocones is required to avoid photons being totally reflected.

\subsection{4: A General Overview of the different Geometrical Structures}

From the previous results, it is apparent that the critical angle plays an extremely important role in current output and efficiency of the solar cell. Figure 4.1.4 also shows that the geometry has a great influence on the critical angle, which too determines the current output and efficiency of the cell. The dependency the two have on each other allows for balance to be created between the height and the critical angle, which will result in the optimal design for light trapping. From Figure 4.1.4, the nanopillar surface design with a height of $60 \mathrm{~nm}$, was shown to have the ideal balance between height and critical angle. There is also a geometrical dependency on the nanostructure, which directly effects what the critical angle of the cell will be. The critical angle allows for the most effective wavelengths of light to be trapped by the cell, which in turn, increases its overall output current. In addition to this, the geometry must be considered when accounting for the height of the structure as different geometrical properties 
will therefore yield a different critical angle ratio, which directly determines what the current output of the solar cell is.

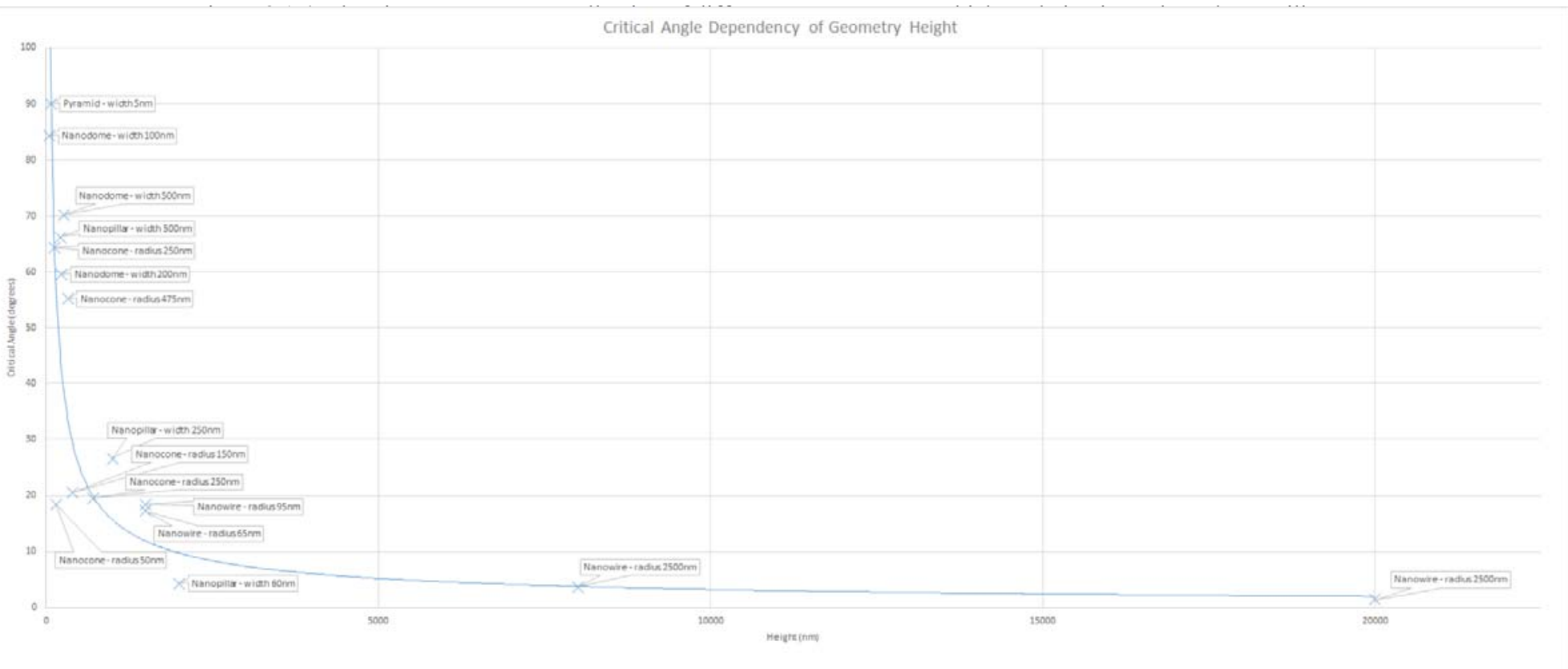

\subsection{1: The étendue of ratio of different nanostructures}

The data points on the graph can be used to directly compare the étendue ratio and thus compare the entropic losses between each of the surface nano-structures.

Geometry plays a large role in the overall efficiency of the cell. The angle at which light enters the cell determines if it will perform any useful work, and by altering the structure to increase the chances of useful work. Smaller distances between disturbances enhances the scattering of light, achieving resonance for a particular wavelength of light and therefore increasing the energy output of the cell.

It is apparent from the plotted graph that the étendue matching of the nanocones with a periodicity of $350 \mathrm{~nm}$ is the most promising candidate structure. It is also visible that the open circuit voltage is greatly affected by the structural properties of the material and that étendue matching increases as the open circuit voltage increases. The importance of étendue matching is that it decreases photon loss during the operation of the solar cell. As aforementioned, étendue is a thermodynamic description of entropy and adds to a decrease in efficiency. Since the ratio of étendue incoming and outgoing is taken as the logarithm it exhibits an exponential dependency on the nanostructures used. 


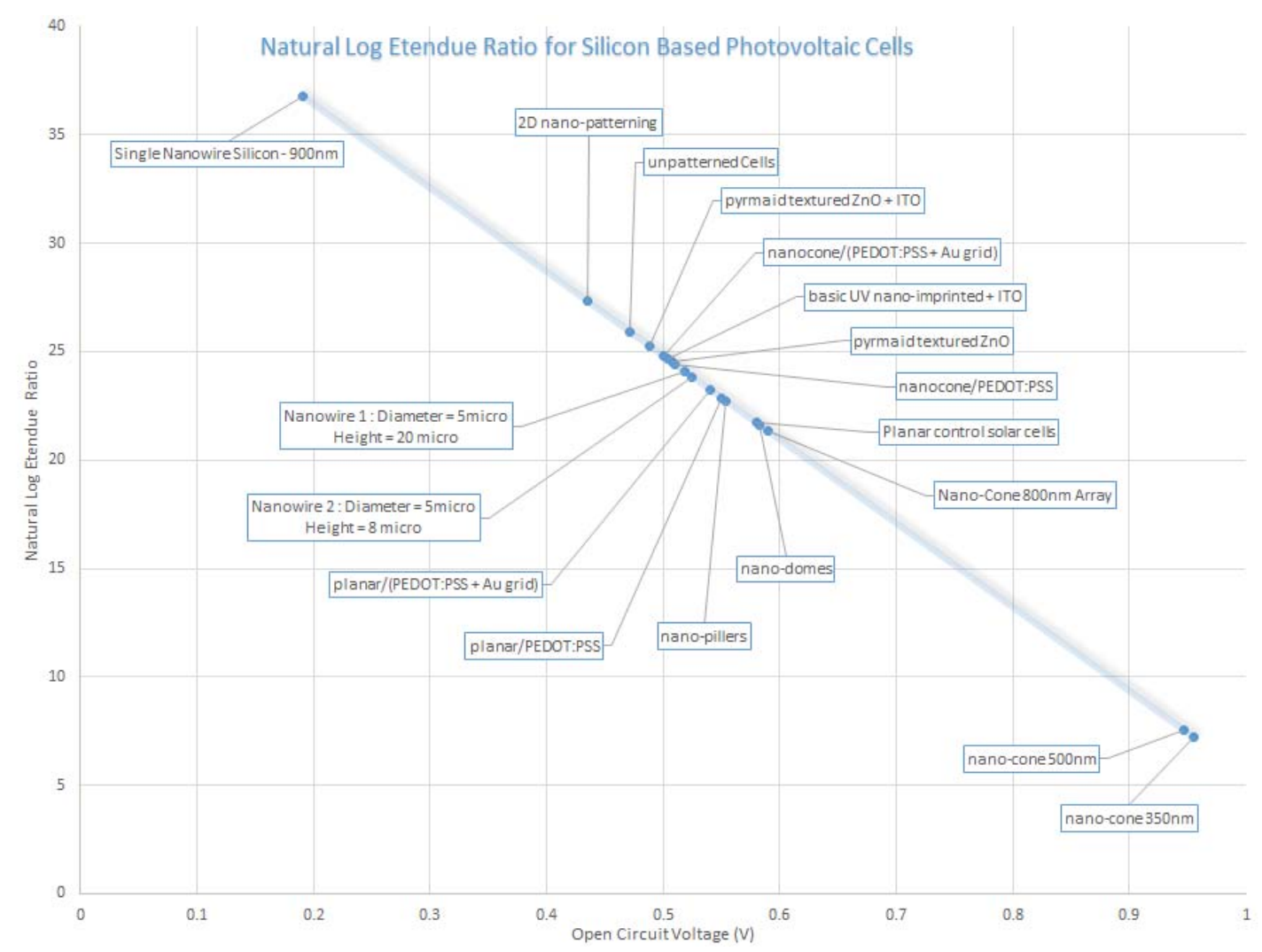

Figure 3.2.1. The Figure represents the ratio of the étendue of each nanostructure using the detailed balance limit equation introduced in the Shockley Queisser section. The ratio is the étendue outbound over étendue incident. A ratio of 1 results in zero light loss [25, 30, 35, 36, 37]

\section{Discussion:}

From the results section, it is apparent that height is a big factor in the determination of the critical angle. The importance of the critical angle is that it prevents the incident photons from escaping the light trapping nanostructure and hence, more electrons can be generated from high energy photons. It is also apparent that the smaller the critical angle the more current can be generated from the nanostructure, which can then be used to do useful work. The factor which also effects the critical angle as well as the height of the structure, is the surface area. The critical angle is not dependent as such on the geometry, but the surface area is directly affected by the geometric structure. The current produced by a geometry is linearly dependent on the surface area therefore it is vitally important to construct a geometric structure which exhibits a very large surface area, as to promote photon absorption. From section 3.1.2 it is evident that the critical angle has an immense impact on the produced current. This was accounted for, so that a smaller critical angle reduces the probability of a photon escaping the structure.

Since light trapping geometries are of vital importance in trapping high energy photons, a careful selection in the semiconductor material plays a huge role in the absorption of the incident photons. From the black body section, the spectrum and intensity of the photons is determined by the temperature of the sun. From the Planck distribution, it can be seen that the intensity demonstrates a definite peak at a certain wavelength. It is therefore important to use a semiconductor material which can absorb photons 
at the wavelength peak. The reason for this being that the spectrum of the body is being fully employed and converted to useful energy. Therefore, it is vital to trap the photons which can promote electrons across the bandgap in the nanostructure.

Another factor which was found to affect the light trapping of solar cells is the Shockley Queisser detailed balance limit which dictates that the fluxes of the incoming and outgoing photons have to be equal. The outgoing flux is a result of the recombination of the electrons with holes which produce a photon that can escape the solar cell. Hence, choosing an appropriate semiconductor combined with a geometrical optimized structure would enable the solar cell to convert the sun's emitted photons into useful electrical energy. A careful inspection of figure 1.1. demonstrates a peak in the spectrum of the sun where the intensity (number of photons) is at its maximum. This region of the spectrum should be fully employed by an appropriate semiconductor material.

The light trapping of solar cells is also susceptible to another fundamental limit, namely the Yablonovitch limit. As discussed in the introduction, the Yablonovitch limit is valid in the realm of structures which encompass structures which are greater than the incident wavelength. This has been demonstrated experimentally in the literature review where the current generated closely follow the imposed limit. This further demonstrates that solar cells are limited in the sense of fundamental thermodynamical limits. The greatest implication which solar cell architecture has to face is the ability to reduce the parasitic étendue increase in the optical system. Formally étendue can increase which demonstrates photons being lost as they propagate through the medium. This is comparable to other energy systems where entropy generation is one of the greatest challenges.

There is a trade off in the ability to minimize the nanostructure height and the achieved low critical angle. From Figure 3.1.4 it can be seen that a nanopillar with a diameter of $60 \mathrm{~nm}$ is in the perfect region where the structure both exhibits a low critical angle combined with a moderate structure height. However, the étendue ratio of the nano pillars is moderately high which increases the amount of light being lost during its propagation in the light trapping scheme. Nanocones however appear to have a very low étendue ration which correlates to a low photon loss within the solar cell structure. This difference in the étendue ratio is quite large when comparing the nanocone to the nanopillars. It is therefore important to develop a geometry which incorporates elements of both geometries.

\section{Conclusion}

It was found that the efficiency of the solar cell is heavily dependent on the ability to capture and trap photons. It was also found that efficiency is greatly influenced by the surface geometry and the area of the nanostructures in the solar cell. Several limitations have been placed on solar cells one of which is the Yablonovitch limit, which interprets the effective path travelled by the photon in a thermodynamic sense. However, from the literature, it is apparent that this limit takes its validity in the microstructure region, where the structures are in the order of several wavelengths. The other limitation imposed on the energy conversion of photons in solar cells is called the Shockley Queisser limit which demonstrates 
that the promotion of an electron to the conduction band is a completely probabilistic event and can be increased with the use of light trapping schemes.

The main aim of light trapping is to increase the probability of high energy photons being absorbed by the semiconductor material to promote electrons. These photons are required to have an energy equal to or greater than the semiconductor bandgap. From the collected data is was found that the Nanopillars show the greatest surface area factor compared to all the other geometries. However, the current produced by the nanocones seemed to be of greater magnitude. It is therefore critical to manufacture a nanostructure which incorporates both a high current density and a large effective area. Furthermore, for nanowires, it was found in the meta study that there is a linear correlation between the area and the output current generated by the geometry. The consequence of this finding is that there is no imposed limit on the height of the structure in regards to its light trapping capabilities.

The light trapping geometries, which exhibit a small $\alpha$ angle, also were found to generate a greater current, due to the ability to establish a Fabry Perot reflection and an increased scatter effect of the photons. This is perfectly demonstrated by the nanowire, where the $\alpha$ angle exponentially decreases with height. The importance of light scattering in the geometry is due to the increased probability of a photon being absorbed by the material and hence, promoting more electrons to the conduction band, which can be used to generate current. Additionally, a smaller $\alpha$ angle imposes a probabilistic barrier for photons to escape the light trapping structure, which means that recombination events do not cause a loss in photons. This also aids in the minimization of étendue formation, which is formally described as light loss in the optical devices. Nanostructures, such as nanopillars, Nanowires, nanocones, and TCOs have shown to be promising candidates in optimizing the efficiency of photovoltaic cells and aiding in the minimization of the Étendue ratio.

\section{References}

1. Markvart, T. Solar cell as a heat engine: energy-entropy analysis of photovoltaic conversion. Phys.Stat.Sol.(A), 2008, Volume 205, pp.2752-2756. Available online: doi: http://dx.doi.org/10.1002/pssa.200880460 (accessed on 13.05.2016)

2. Giancoli, D.; Eibl, O. München: Pearson Studium. Physik, 2010, pp. 707-714; 1267

3. Hirst, L.; Ekins-Daukes, N. Fundamental losses in solar cells. Prog. Photovolt: Res. Appl, 2010, Volume 19, pp. 286-293. Available online: doi: http://dx.doi.org/10.1002/pip.1024 (accessed on 13.05.2016)

4. Rau, U.; Paetzold, U.; Kirchartz, T. Thermodynamics of light management in photovoltaic devices. Phys. Rev. B, 2014, Volume 90. Available online: doi: http://dx.doi.org/10.1103/PhysRevB.90.035211 (accessed on 13.05.2016)

5. Lemons, D. Mere thermodynamics. Baltimore: Johns Hopkins University Press, 2009, pp. 64-68; 101-104

6. Feynman, R.; Sands, M.; Leighton, R. A Member of the Perseus Books Group. The Feynman lectures on physics, 2010, pp. 41-7;41-8; 44-10; 44-11; 44-12 
7. McDonald, K.T. Brightness and Etendue of an Aperture Lamp. Intensity, 2013

8. Atkins, P.; De Paula, J. Elements of physical chemistry, Oxford: Oxford University press, 2013, pp. 90-96

9. Atkins, P.; De Paula, J. Physical chemistry, Oxford: Oxford University press, 2014, Volume 10, pp. 114-117

10. Markvart, T. From steam engine to solar cells: can thermodynamics guide the development of future generations of photovoltaics?. Wires Energy Environment, 2016, Available online: doi: http://dx.doi.org/10.1002/wene.204 (accessed on 13.05.2016)

11. Krogstrup, P.; Jørgensen, H.; Heiss, M., Demichel, O.; Holm, J., Aagesen, M., et al. Singlenanowire solar cells beyond the Shockley-Queisser limit. Nature Photonics, 2013, Volume 7, pp.306-310. Available online: doi: http://dx.doi.org/10.1038/nphoton.2013.32 (accessed on 13.05.2016)

12. Mellor, A.; Tobías, I.; Martí, A.; Mendes, M.; Luque, A. Upper limits to absorption enhancement in thick solar cells using diffraction gratings. Prog. Photovolt: Res. Appl., Volume 19, pp. 676-687, Available online: doi: http://dx.doi.org/10.1002/pip.1086 (accessed on 13.05.2016)

13. Wood, M. Etendue. Out Of The Wood, 2012

14. Wehrspohn, R.; Üpping, J. 3D photonic crystals for photon management in solar cells. Journal Of Optics, 2012, Volume 14 Available online: doi: http://dx.doi.org/10.1088/2040$\underline{8978 / 14 / 2 / 024003}$ (accessed on 13.05.2016)

15. Markvart, T. The thermodynamics of optical étendue. Journal Of Optics A: Pure And Applied Optics, 200, Volume 10, Available online: doi: http://dx.doi.org/10.1088/1464-4258/10/01/015008 (accessed on 13.05.2016)

16. Zhu, H.; Blackborow, P. Etendue and Optical Throughput Calculations, ENERGETIQ, 2011

17. Trupke, T.; Green, M.; Würfel, P. Improving solar cell efficiencies by down-conversion of highenergy photons. J. Appl. Phys., 2002, Volume 92

18. Shockley, W.; Queisser, H. Detailed Balance Limit of Efficiency of p-n Junction Solar Cell. J. Appl. Phys., 1961, Volume 32, pp.510, Available online: doi: http://dx.doi.org/10.1063/1.1736034 (accessed on 13.05.2016)

19. Polman, A.; Atwater, H. Photonic design principles for ultrahigh-efficiency photovoltaics. Nature Materials, 2012, Volume 11, pp.174-177. Available online: doi: http://dx.doi.org/10.1038/nmat3263 (accessed on 13.05.2016)

20. Markvart, T. Thermodynamics of losses in photovoltaic conversion. Appl. Phys. Lett., 2007, Volume 91, Available online: doi: http://dx.doi.org/10.1063/1.2766857 (accessed on 13.05.2016)

21. Ulbrich, C.; Fahr, S.; Üpping, J.; Peters, M.; Kirchartz, T.; Rockstuhl, C. et al.Directional selectivity and ultra-light-trapping in solar cells. Phys. Stat. Sol. (A), 2008, Volume 205, pp.2831-2843,

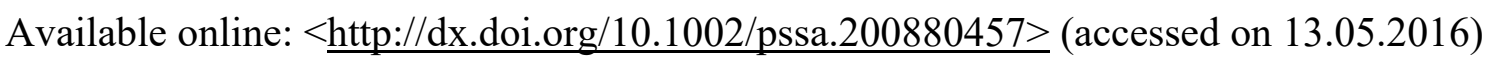

22. Yablonovitch, E. Statistical ray optics. Optical Society of America,Volume 7, 1982

23. Fonash, S. A Brief Overview of Phenomena Involved in Light Trapping. Light Trapping in Solar Cell and Photo-Detector Devices, 2015 
24. Yu, Z.; Raman, A.; Fan S. Fundamental Limit of Nanophotonic Light-trapping in Solar Cells. Proceedings of the National Acadamy of Sciences of the United States of America, 2008, Volume 107

25. Garnett, E.; Yang, P. Light Trapping in Silicon Nanowire Solar Cells. Nano Letters, 2010, Volume 10, pp.1082-1087

26. Wallentin, J.; Anttu, N.; Asoli, D.; Huffman M.; Åberg, I.; Magnusson, M.H.; Siefer, G.; FussKailuweit, P.; Dimroth, F.; Witzigmann, B. InP Nanowire Array Solar Cells Achieving 13.8\% Efficiency by Exceeding the Ray Optics Limit. Science, 2013, Volume 339

27. Anttu, N.; H. Q.; Xu, J. Coupling of Light into Nanowire Arrays and Subsequent Absorption, Nanosci. Nanotechnol., 2010, Volume 10

28. Kupec, J.; Stoop, R.L.; Witzigmann, B. Light Absorption and Emission in Nanowire Array Solar Cells. Opt. Express, 2010, Volume 18

29. Wadhwa, P.; Liu, B.; McCarthy, M.A.; Wu, Z.; Rinzler, A.G. Electronic Junction Control in a Nanotube-Semiconductor Schottky Junction Solar Cell, Nano Letters, 2010

30. Wong, S. M.; Yu, H.Y.; Li, J. S.; Zhang, G.; Lo, P.G.Q; Kwong, D.L. Design High-Efficiency Si Nanopillar-Array-Textured Thin-Film Solar Cell. IEEE Electron Device Letters, 2010, Volume 31

31. Xingze, K.; Wang, Yu, Z.; Liu, V.; Cui, Y.; Fan, S. Absorption Enhancement in Ultrathin Crystalline Silicon Solar Cells with Antireflection and Light-Trapping Nanocone Gratings. Nano Letters, 2012

32. Brongersma, M.; Atwater, H.; Polman, A. Nanostructures for Light Trapping in High-efficiency, Low-cost Silicon Solar Cells. Global Climate \& Energy Project, Stanford University, 2013

33. Fan, Z.; Kapadia, R.; Leu, P.W.; Zhang, X.; Chueh, Y.; Takei, K.; Yu, K.; Jamshidi, A.; Rathore, A.A.; Ruebusch, D.J. Ordered Arrays of Dual-Diameter Nanopillars for Maximized Optical Absorption. Nano Letters, 2010

34. Springer, J. Light trapping and optical losses in microcrystalline silicon pin solar cells deposited on surface-textured glass/ZnO substrates. Solar Energy Materials and Solar Cells, 2004

35. Jeong, S.; Garnett, E.; Wang, S.; Yu, Z.; Fan, S.; Brongersma, M.; McGehee, M.; Cui, Y. Hybrid Silicon Nanocone-Polymer Solar Cells. Nano Letters, 2010

36. Hsu, C.; Battaglia, C.; Pahud, C.; Ruan, Z.; Haug, F.; Fan, S.; Ballif, C.; and Cui, Y. High-Efficiency Amorphous Silicon Solar Cell on a Periodic Nanocone Back Reflector. Advanced Energy Materials, 2012, Volume 2

37. Battaglia, C.; Söderström, K.; Escarré, J.; Haug, F.; Dominé, D.; Cuony, P.; Boccard, M.; Bugnon, G.; Denizot, C.; Despeisse, M. Efficient light management scheme for thin film silicon solar cells via transparent random nanostructures fabricated by nanoimprinting. Applied Physics Letters, 2010, Volume 96

38. Muller, J.; Rech, B.; Springer, M. TCO and light trapping in silicon thin film solar cells. Science Direct Online, 2004, pp. 917-930

39. Zhu, J.; Hsu, C.; Yu, Z.; Fan, Z.; Cui, Y. Nanodome Solar Cells with Efficient Light Management and Self-Cleaning. Nano Letters, 2010

40. Bockmeyer, M.; Schmidt, F.; Rech, B. Large-area 2D periodic crystalline silicon nanodome arrays on nanoimprinted glass exhibiting photonic band structure effects. ResearchGate: Nanotechnology, 
2012. Available online: https://www.researchgate.net/publication/221709309 (accessed on 13.05.2016)

41. Kelzenberg, M.; Turner-Evans, D.; Kayes, B.; Filler, M.; Putnam, M.; Lewis, N.; Atwater, H. Photovoltaic Measurements in Single-Nanowire Silicon Solar Cells. Nano Letters, 2008, Volume 8.

42. Zhao, J.; Wang, A.; Campbell, P.; Green, M. 19.8\% Efficient Honeycomb Multicrystalline Silicon Solar Cell with Improved Light Trapping. IEEE Transactions on Electron Devices, 2002, Volume

43. Oh, J.; Yuan, H.; Branz, H. An 18.2\%-efficient black-silicon solar cell achieved through control of carrier recombination in nanostructures. Nature Nanotechnology, 2012, Volume 7

44. Zolper, J.; Narayanan, S.; Wenham, S.; Green M. 116.7\% efficient, laser textured, buried contact polycrystalline silicon solar cell. Applied Physics Letters, 1989, Volume 55

45. Trompoukis, C.; Daif, O.; Depauw, V.; Gordon, I.; Poortmans, J. Photonic assisted light trapping integrated in ultrathin crystalline silicon solar cells by nano-imprint lithography. Applied Physics Letters, 2012, Volume 101

(C)2016 by the authors. This article is distributed under the terms and conditions of the Creative Commons Attribution 4.0 International License (http://creativecommons.org/licenses/by/4.0/). 